\title{
THE PERMIAN END CATASTROPHE - AN EVIDENCE FOR A BROADER PHENOMENON
}

\author{
Sergio Meth \\ Universidade Federal do Pampa - Campus Bagé, RS, Brasil \\ sergiometh@gmail.com
}

\begin{abstract}
It is well known that about 250 million years ago a huge catastrophe took place in our planet, with effects so big that about $90 \%$ of the species disappeared in the process including sea animals. This event is called "the Permian extinction". Other effects are also apparently connected to this event. This kind of event would require an extremely huge amount of energy. Since there are no evidences for an asteroid impact, the energy source should be searched inside the planet. Most important, it should show an energy source capable of producing such huge phenomenon. The aim of this paper is to propose a model to explain this extinction event and to show that there are evidences that this is a phenomenon that apparently happened several times not only in our planet but also in other Solar System bodies.
\end{abstract}

Keywords: Permian extinction; geo-physical-chemistry; nuclear energy 


\section{INTRODUCTION}

The aim of this paper is to propose a model to explain the so called Permian extinction and to show that there are evidences that this is a phenomenon that apparently happened several times not only in our planet but also in other Solar System bodies.

It is well known that about 250 million years ago a huge catastrophe took place in our planet, with effects so big that about $90 \%$ of the species disappeared in the process including sea animals. This event is called "the Permian extinction". There is no evidence supporting the possibility of an asteroid crash like the one that, 65 million years ago, ended the dinosaurs' era. Other effects are also apparently connected to this event. The most important being perhaps the Earth's crust cracking giving the start to the so called Continental Drift. The best explanation up to this moment is that a broad phenomenon took place in a very large area in Siberia with a big lava flow, today known as "Siberian Traps". Together with this lava flow a huge release of noxious gases took place causing this big extinction. The geological evidence shows the connection between two of these facts: the species extinction and the Siberian Traps. However the crust cracking needs a better explanation since this kind of event would require an extremely huge amount of energy. Since there are no evidences for an asteroid impact, the energy source should be searched inside the planet. Most important, it should show an energy source capable of producing such phenomenon on a planet scale.

\section{The geological evidence}

The amount of species extinct in the Permian End, 250 million years ago, is so huge that only an extreme catastrophe in planetary scale can explain such life holocaust. The estimates are that about $90 \%$ of all species disappeared. Even very successful species like the trilobites, arthropods that lived in the ocean and lasted for at least 300 million years, were gone. The trilobites lived through the whole Paleozoic Era, englobing six geological periods (Cambrian, Ordovician, Silurian, Devonian, Carboniferous and Permian). They can be considered as one of the more successful animal order, if not the most successful, with more than 20.000 species, found in the geological record, have already been described in the literature. It is important to emphasize that such large 
numbers of species extinction in this event (Permian End) would mean that life almost ended on Earth.

There is a consensus in the science community that the best explanation is the so called Siberian Traps that took place exactly in the Permian end period. This event is shown in the geological record as massive volcanic eruptions in a very broad area in Siberia that expelled more than 4 million cubic kilometers of lava. This event would also have expelled such tremendous amounts of noxious gases into the atmosphere, with consequences in the oceans, practically making life almost impossible in the whole planet. Only some forms of life were capable of surviving the resulting harsh conditions.

The Permian period, like other geologic periods, is a time span where there were no big changes in the ecological environment. And suddenly a completely new environment appears. The Permian lasted for about 48 million years. Considering the Paleozoic Era as a whole, each period lasted about the same average period of time (48 million years) with some differences from one period to another. This number is particularly important for considerations late in this paper.

A question remains: what caused the Siberian Traps? The previous periods, Carboniferous and Permian, don't show any evidence of big volcanic activity, by the contrary, it is very apparent that these periods had a relative calm in the geological activity and this is very well demonstrated by the stability in the ecologic environment. This would mean that inside the planet also the convection flows were relatively calm during these two periods lasting about 100 million years. Whatever caused the Siberian Traps released from within the planet a tremendous amount of energy if only this event is considered.

However the planet crust cracking also took place parallel to the Siberian Traps. Until the Permian end there are few apparent evidences of the so called Continental Drift, if any. The crust cracking that led to the nowadays continents and others, like Australia and India, apparently happened together or very close associate in time to the Siberian Traps. Also for the Continental Drift to be kept afterwards for such a long time span would require the planet internal convection currents with such strength that seems to not be present so strong in the Carboniferous and Permian periods. Also this surface cracking would require such an amount of energy difficult to imagine. 
An interesting point to observe involving the cracking event is that the so called Pacific Plate is very big and has an almost round shape. It looks like a ball shadow with a much defined border and an apparent stable interior, without volcanic and seismographic activity inside and a lot in the border of the plate. In contrast there was a lot of cracking in the rest of the planet surface.

Taking the two facts, the Siberian Traps, and the planet crust cracking, it is difficult not to associate the two events. Most important, both would require a sudden tremendous energy release. Since such kind of release probably is unique over a long time spam, it is not unreasonable to consider that they took place at the same time. The solid confirmation that these events were parallel in time by field research would be a very strong proof that they happened due to the same cause.

It is necessary also to consider the movement of the magnetic poles relative to the geographic ones. The geological evidences show that there is an irregular pattern. Since the magnetic field in the planet is caused by the spin of a solid core (composed basically by Iron and Nickel), the modification of the magnetic poles' axis suggest a change in the planet solid core spinning. Also this would require high amount of energy, particularly to change the direction of the axis as shown in the geological record. However this is not a very clear fact since these modifications in the magnetic poles happened and happens continuously over time. If it is possible to correlate the periodic life extinction and the sudden change in the movement of the magnetic poles' axis is a question that could corroborate the correlation between the sudden energy release from within the Earth and the other events here describe like the Permian Extinction.

\section{The astronomical evidences}

The Solar system is supposed to have been formed from a rotating dust cloud in the shape of a flat dish. It is very important to consider the angular moment conservation to explain the facts as they present to us nowadays. The planets make a translation movement around the sun all in the same direction and roughly in the same plane with small deviations. Also the rotation movement by most of the planets is in the same direction. By the Earth point of view it is counterclockwise if the planet is observed above the North Pole, or in other words, the planet moves towards the East. The sun rotates itself also in the same direction. The plane of these movements is called Ecliptic 
and it is possible to see it in the sky simply because it passes through the zodiac constellations. This plane and the same direction of these movements seem feasible since the angular moment is to be conserved. The planets rotation axis should have a $90^{\circ}$ angle to the Ecliptic Plane if the angular moment was supposed to be conserved. However some of the planets have an angle difference in the rotation movement, including Earth. What caused this difference? Also here it would be necessary a huge, tremendous amount of energy to cause this change and also it would be necessary to have a compensation somewhere to conserve the angular moment.

Confirming the rule our Moon translates around the Earth and also rotates itself, both movements roughly in the ecliptic plane, with a small deviation of about $5^{\circ}$. The best accepted theory considering the moon formation is that a huge body, same size as Mars, shocked with our planet and from this crash there was a formation of a ring cloud that over time coalesced and formed the moon. It is reasonable to consider that the movement of this huge body had to be on the Ecliptic Plane since the resulting cloud and for consequence the Moon remained on the same plane. But if the Earth rotation axis would be already out of $90^{\circ}$ angle from the Ecliptic Plane by the time of the crash, the moon formation cloud would not be so close to the Ecliptic Plane due to the angular moment conservation. This conclusion can be applied to Moon movements, translation and rotation, both with a rotation axis roughly close to a $90^{\circ}$ angle from the Ecliptic Plane. It seems reasonable to consider by these evidences that the Moon, or at least the cloud that originate it, was formed before the Earth rotation axis was tilted. But to do such a difference in the rotation axis would require enormous amounts of energy and there is no evidence of another big crash with a huge celestial body that would cause this change in Earth axis rotation.

It seems reasonable to consider the Permian end event and the Earth axis tilt as similar in the sense that both would require that big amount of energy. The tilt axis event doesn't show scars on Earth surface nowadays probably because the crust was thin by the time that this event took place and the effects on the surface would have been obliterated over time by the geologic activity on the surface. But by the time of the Permian end the crust was already enough thick to keep the "scars" that in the end reshaped our planet dividing the only continent in several ones. Once the crust was cracked, the internal convection forces made their work separating each time more and more the so divided new continents. 
There are these facts in our Solar system that need a better explanation. The tilt angle deviations from the Ecliptic Plane that exists in our and some other planets rotation axis is only one. The Saturn rings in the same tilted rotation plane of rotation of this planet suggests that they were formed in the same event that tilted the rotation axis of the planet. The planet that should exist between Mars and Jupiter and it is not there. In its place there are irregular objects called in the literature asteroids that are considered by some theories as resulting from an explosion of this "proto planet". Although some theories say that such planet was never formed, the irregular shapes of these bodies suggest that they were formed in a disintegration of a bigger body caused perhaps by an internal energy release. The huge variation of the angles of the orbit of these bodies around the Sun to the ecliptic plane can corroborate the explosion explanation. Destruction by Jupiter's gravity or even if because of this reason the planet was not formed would have resulted in orbits on the Ecliptic plane. Also the cracking on the surface of Europa, a Jupiter moon, can be explained based in the idea of a huge internal release of energy.

Looking at the big picture not only in our planet but also in other celestial bodies, the idea of a sudden enormous energy release from within seems very feasible. Them the question is: what can be the cause of this phenomenon?

\section{The internal planet source of energy}

Considering the facts described, it seem reasonable to accept that the shock with celestial bodies are not the only explanation for the events that are in the geologic and astronomic record. The moon's formation and the dinosaurs' extinction were clearly caused by such kind of crash, but for many other events there are no evidence of a celestial crash. By the contrary, the evidences suggest some kind of energy release from within the planet. This is probably by all the facts presented the cause of the Permian extinction.

Any explanation for an internal release of energy should show why there are periodic huge extinctions in the geological record. Considering only the Paleozoic Era, there is an average of about 50 million years for the span time between each extinction with some variability: Cambrian - $54 \mathrm{My}$, Ordovician - $44 \mathrm{My}$, Silurian - $28 \mathrm{My}$, Devonian - 57 My, Carboniferous - 60 My, Permian - 48 My (My - millions of years). Such 
regularity suggests some kind of "Nature clock". Since some of the events are not connected to celestial events, it is necessary to present facts from within the planet.

Since the Nineteenth Century it is very well known that without an internal heat source the inner part of the Earth planet would have cooled down very fast. Initially a short period was calculated, by the order of hundreds of thousands of years, for the cooling and this was considered as an indication for an Earth dating ruler in spite of not fitting with the geological records. During the Twentieth Century it became clear that Earth keeps the interior hot by the disintegration of radioactive elements such as Uranium and Thorium. These elements would be found in the primordial cloud that gave birth to the Solar system due to the fact that this cloud was formed by the explosion of one or more supernovas. In such events, elements heavier than Iron are formed, including the radioactive ones. In a "normal" star like the Sun, only elements up to Iron are formed.

The geologic accepted model is that the radioactive disintegration of these elements is not fast, just keeping the planet in a steady state, with the heating being the source of the so called convection flow. The convection flow would be the cause of the Continental Drift. This model seems very feasible and in fact is very attractive. There is practically a consensus about it. If a static situation is considered like in the present moment, with earthquakes and volcanic activity connected to events close to the crust surface due to the convection internal forces, the model is in fact very convincing.

The elements considered in this "internal geologic radioactive model" have high relative density when compared with other elements: Thorium $=11,8$; Uranium $=19,1$; Neptunium $=20,5$; Plutonium $=19,9$. The oxides, particularly the Uranium Oxide, also have high density. In comparison, Iron and Nickel have densities of 7,9 and 8,9 respectively.

Only Thorium and Uranium are both radioactive and "abundant". Neptunium and Plutonium have half-lives so short that can be found only in trace amounts mostly of them produced by decay or nuclear reaction of Thorium or Uranium. It is considered that the core inside Earth is composed mainly by Iron and Nickel and it was formed due to the density of these elements that went to Earth center. It seems reasonable to think that the same effect would happen to the radioactive elements, mainly to Uranium, due also for the higher density of this element. If this is considered, it is also reasonable to accept that over time particularly Uranium would be concentrating close to the "solid core". The affinity of Uranium and Silica would decrease the speed of the Uranium 
towards the center and the increase of the Uranium concentration in comparison to Iron and Nickel would be slower because the Uranium would be more locked to the bulk material. The convection flow, with lava going up, down and even to the sides, would also be a disturbing fact, but overall in the average the Uranium would go little by little towards the Earth center, stopping in the border of the "hard core", remaining and concentrating in this area.

This slow movement to the center would be in fact very, very slow. If we consider a sphere of $12,500 \mathrm{Km}$ of diameter, as a whole, without a crust or a core, with an average of $10 \mathrm{~cm}$ a year, a given atom of Uranium would take 62.5 million years to go from the surface to the very center of the planet. These numbers are in the same magnitude order of the extinction intervals of time, as said before, around 50 million years.

What was the amount of Uranium in the primitive solar system cloud? This is a very important fact but it seems very elusive to be determined with a good precision. Anyway, this concentration would be connected to the Uranium concentration in primitive Earth, but how was the dynamics of the concentration phenomenon in the beginning, how was the effect of the crash with the Mars size body, etc, etc? It is in fact a very difficult number to be estimate. For sure the information about the Earth interior is far from being conclusive nowadays. The information about the liquid interior and the core is not direct but the ones given by secondary effects like shock waves from earthquakes.

If the average concentration of $0.0003 \%$ of Uranium in Earth crust is applied to the entire mass of the planet $\left(6 \times 10^{24} \mathrm{Kg}\right)$, it is calculated that the total amount of Uranium would about $1,800,000$ Tons. However, probably the concentration in the planet interior is bigger due to the geologic process over a very long period based in the Uranium higher density. Another aspect is that the amount of Uranium goes down over time due to radioactive decay. Everything taken together is not unreasonable to say that the amount of Uranium 4.6 billion years ago (Earth beginning) would be bigger than nowadays, and that in earth center there is much more than the amount that the crust Uranium concentration revealed by the field studies.

The main Uranium isotopes are $U^{235}$ and $U^{238}$, with an abundance of $0.72 \%$ of the 235 isotope. Since the most important isotope for use (by human technology) is the 235, this should be concentrate. Atomic reactor or atomic bombs are the main uses of Uranium with higher 235 concentration. For comparison the bomb used in Hiroshima had an 
explosive capacity of $15 \mathrm{Ktons}$ of TNT and used $65 \mathrm{Kg}$ of $\mathrm{U}^{235}$. Considering the $0.72 \%$ of the 235 isotope for 1,800,000 Tons of Uranium, it would mean that the total of the Uranium 235 in Earth would be around 13,000 Tons, or the equivalent to 200,000 Hiroshima bombs or 3,000,000 Ktons of TNT. This number is probably much bigger, mainly inside Earth, and is difficult to estimate it for sure.

The Uranium being concentrated close to the core would be submitted to a very high pressures and temperatures, supposedly 6,000 $\mathrm{C}$ and 1.4 million atmosphere by the last estimates. Also it would be very difficult to understand by any model or experiment how is the behavior of this material in these conditions. For the human technology it is necessary to concentrate the 235 isotope. But is it necessary to have a $\mathrm{U}^{235}$ concentrate isotope in the Earth center conditions to start a chain reaction? It seems feasible to think that the chain reaction between the $\mathrm{U}^{235}$ isotope could be triggered easier in such conditions even with the low concentration of this isotope considering the Uranium as a whole.

Conditions in nature can be different and it is known that the natural Uranium can produce by itself a "chain reaction" even close to Earth surface. This was demonstrated by the so called "Oklo Phenomenon", a natural occurring nuclear reactor located in Gabon - Africa, where at least six spent natural reactors have "burned" the $U^{235}$ during at least $1.8 \times 10^{9}$ years reducing the amount of the $U^{235}$ isotope to $0.296 \%$ (from the initial $0.72 \%$ ). The mine contains about $60 \%$ of Uranium. Water was used as a moderator by nature avoiding a "runway" reaction after the nuclear chain reaction initiated. Anyway it was very well demonstrated that a chain reaction can be sustained by the $\mathrm{U}^{235}$ natural concentration in the Uranium found in nature.

Therefore the Uranium would concentrate little by little from the surface to the center. After millions of years the higher Uranium concentration close to the core, not entering it since the solid nature of the very center core, being submitted to the very high temperatures and pressures would not only start a "controlled" chain reaction but would also have gathered the fissional materials in such a huge amount in a state that could be described as "almost explosive nuclear gel" ready to start a very huge nuclear chain reaction explosive event. Any event, like the shock waves from an earthquake close to the surface could perhaps trigger such explosion and, depending on the amount of material gathered, the energy release would be enough to cause the effects described 
previously. The main proof of this being the Permian end event, but other events in the geologic and astronomic records can be also considered.

In this model it is possible so to understand how takes place the sudden energy releases that happen from time to time. Not only the extinction and cracking crust effects are to be considered, but also the planet tilt axis should be taken into consideration. The angular moment conservation would be kept by the resulting convection magma flow inside the planet. This would explain very well the increase of the geological activity after the Permian end event.

\section{Final considerations about fitting the model with facts and events}

The Permian end event can be explained by a huge explosion within the planet caused by the concentrated Uranium. This explosion would have triggered the Siberian Traps, caused shock waves that would have cracked the Earth's crust and probably caused by itself a lot of destruction on planet's life by the shock wave even before the effects of the Siberian Traps could be felt. The crust separated blocks would remain as scars of this explosion with the Pacific Plate being the shadow of the core and therefore this area was the less affected by the explosion.

If it is considered that the rotation axis is tilted, it is indicating that a similar event took place in the very beginning of the planet but after the Moon's formation, or at least the dust cloud that originated the Moon.

The periodic extinctions can be explained by this model by a repetition in a small scale, when compared to, of the Permian end explosion. Such internal events would trigger high volcanic activity and would affect life on a planetary scale. The time spam can therefore be explained because of the Uranium concentration speed towards the center and by the conditions in the liquid part of the planet. The Carboniferous and Permian, being "calm periods", would also have prepared the planet for the Permian end drama. The inclinations of the rotation axis of the planets could be explained by this kind of event. It is interesting to observe that Mercury and Moon, both relatively small bodies, have no inclination. Is it possible that such small size wouldn't allow the conditions for the inner nuclear explosions? Saturn has a big deviation and rings in the same rotation plane. Would it be possible that a similar event in a gaseous planet would trigger also a thermonuclear reaction? Given the high amount of Hydrogen, why not? Jupiter has a 
small deviation, but it also has a very big size and a lot of mass. Would it be possible that events like the red spot on Jupiter are being triggered by the model here describe. Same comparisons could be made for the other planets. Probably the events in Venus were extraordinary since the planet was turned upside down. Perhaps part of the violent nature of this planet is connected to this, including volcanism and release of $\mathrm{CO}_{2}$ to the atmosphere.

The absent fifth planet could be explained by having faced such a big explosion that in the end destroyed the whole planet leaving asteroids as the astronomic record. Perhaps this planet was big enough to trigger the nuclear chain reaction and small enough to be destroyed by it.

The observed cracking on the Europe's surface can also be explained by this model. And so on

In fact when each situation, event or fact is thought using this model, it gives a very easy and simple explanation. It happens to be so simple that perhaps the situations that are not explained by the shock between celestial bodies can be easily explained using the "Nuclear Energy Sudden Internal Release" model (NESIR).

\section{CONCLUSION}

A model ("Nuclear Energy Sudden Internal Release" - NESIR) is presented explaining several events shown by the geologic and astronomic records. Particularly the evidences connected to the so called Permian end episode. Mathematical modelling, in spite of the limitations given by the absence of information of the Earth interior, will probably help to confirm the model here proposed. Also the model here proposed does not collide with the existing ones but by the contrary confirms them, like the Siberian Trap. Finally this model can be applied to model geological effects linked to important aspects like mining and geological studies. Probably, once confirmed by other evidences that will be uncover, it will help to explain better Earth History and beyond in the Solar System.

Of course, the purpose of this paper is not to give a final and absolute explanation to the described facts sometimes bizarre and of difficult understanding by other models, but mainly to demonstrate that using the proposed model it would be possible to give a better understanding to several facts and events not only from our planet but also in other Solar System bodies. 


\section{BIBLIOGRAPHY}

1. Salgado-Labouriau, M. L.; História Ecológica da Terra, $2^{\text {nd }}$ Ed., Edgard Blucher: São Paulo, 1994;

2. Cloud, P. et al; Adventures in Earth History, W. H. Freeman and Company: San Francisco, 1970;

3. Press, F.; Siever, R., Grotzinger, J.; Jordan, T. H.; Para Entender a Terra, $4^{\text {th }}$ Ed., Artmed Editora S.A.: Porto Alegre, 2007;

4. Popp, J. H.; Geologia Geral, $6^{\text {th }}$ Ed., LTC: Rio de Janeiro, 2012;

5. Teixeira, W.; Fairchield, T. R.; Toledo, M. C. M.; Taioli, F.; Decifrando a Terra, $2^{\text {nd }}$ Ed., Companhia Editora Nacional: São Paulo, 2009;

6. http://www.trilobites.info/, viewed on November 2018;

7. Coney, L. et al; Palaeoworld, 2007, 16, 67-104;

8. Bond, D.; Wignall, P.; Geophysical Research Abstracts, 2018, 20, EGU2018-8134;

9. Greenwood, N. N.; Earnshaw, A.; Chemistry of the Elements, $1^{\text {st }}$ Ed., Pergamon Press: Oxford, 1984. 